\title{
Guest Editorial: Visual Information Processing and Perception
}

\author{
Hari Kalva ${ }^{1} \cdot$ Homer Chen ${ }^{2}$ - Velibor Adzic ${ }^{3}$. \\ Gerardo Fernández-Escribano ${ }^{4}$
}

Published online: 10 October 2015

(C) Springer Science+Business Media New York 2015

The goal of this special issue was to bring together experts working on applying models, principles, and knowledge of human audio-visual perception and cognition to optimize video processing algorithms and applications. This is an area where our deeper understanding of visual perception to video processing will lead to new breakthroughs in visual information processing.

Attentional focus is an important aspect of perception that helps understand user interest and intent. Determining points of saliency is computationally complex. Compressed-domain methods thus become valuable tools for developing computationally efficient and practical solutions. In "Compressed-Domain Correlates of Human Fixations in Dynamic Scenes" (10. 1007/s11042-015-2802-3) authors present a method for detecting points of fixation in H.264/ AVC video using motion vectors, block coding modes and coded residuals parsed from a $\mathrm{H}$. 164/AVC bitstream.

Egocentric videos are captured using wearable cameras and used to detect a wearer's point of view. Amount of video captured using wearable cameras is increasing and saliency detection in such videos enables applications such as efficient video summarization. In "Geometrical Cues in Visual Saliency Models for Active Object Recognition in Egocentric Videos" (10.1007/ s11042-015-2803-2) authors use geometrical cues to improve saliency detection in videos.

Action recognition from videos is a challenging task with many applications including surveillance and social behavior understanding. Inspired by models of neural response to visual input, the paper entitled "Deep Learning Human Actions from Video via Sparse Filtering and Locally Competitive Algorithms" (10.1007/s11042-015-2808-x) presents a method that combines sparse filtering with locally competitive algorithms for applications in action recognition.

Hari Kalva

hari.kalva@fau.edu

1 Florida Atlantic University, Boca Raton, FL, USA

2 National Taiwan University, Taipei, Taiwan

3 Videopura, Delray Beach, FL, USA

4 University of Castilla-La Mancha, Albacete, Spain 
Discovery that user attention can be primed using momentary flashes of light inspired the work presented in "Method and Experiments of Subliminal Cueing for Real-World Images" (10.1007/s11042-015-2804-1) Authors report the use of subliminal cues to retarget attention in images. Such cues can redirect user attention to the target areas that need more attention such as unexpected events in surveillance video.

Neural correlates of visual perception can help understand visual information processing in the brain and enable new class of applications. Neural response, measured using an EEG, is an indicator of user response to visual information processing tasks. In "Improving Object Segmentation by Using EEG Signals and Rapid Serial Visual Presentation” (10.1007/ s11042-015-2805-0) authors show that neural response is strong enough to detect when subjects find target images presented rapidly and that using neural response can improve object segmentation.

Eye movements during a visual performance task have rich information that can be exploited to understand visual perception and user response to the visual stimuli. In "The Influence of Color during Continuity Cuts in Edited Movies: An Eye-Tracking Study" (10. 1007/s11042-015-2806-z) authors report that color aids saccades after continuity cuts. These findings can be applied to more efficient compression of movie videos.

We hope this special issue is able to highlight the potential of applying models of human visual perception in video processing applications. The guest editors would like to sincerely thank the many reviewers that provided valuable input in shaping this special issue.

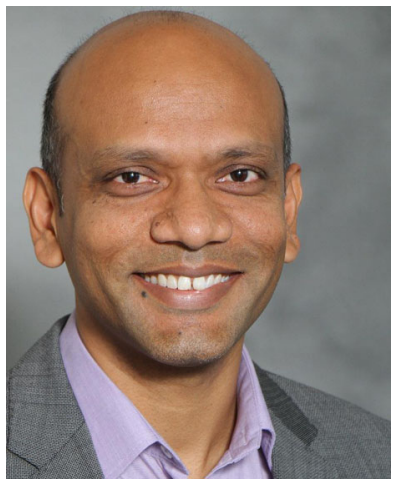

Hari Kalva is a Professor, Associate Chair, and the Director of the Multimedia Lab in the Department of Computer \& Electrical Engineering and Computer Science at Florida Atlantic University (FAU).

Dr. Kalva has over 20 years of experience in multimedia research, development, and standardization. He has made key contributions to technologies that are now part of MPEG-4 standards. His current research focuses on understanding and applying human visual perception, cognition, and social context to optimize visual information processing.

Dr. Kalva received a Ph.D. and an M.Phil. in Electrical Engineering from Columbia University in 2000 and 1999 respectively. He received an M.S. in Computer Engineering from Florida Atlantic University in 1994, and a B. Tech. in Electronics and Communications Engineering from N.B.K.R. Institute of Science and Technology, S.V. University, Tirupati, India in 1991. 


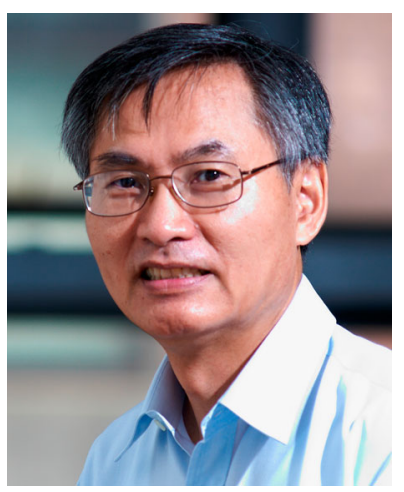

Homer H. Chen (M'86-SM'01-F'03) received the Ph.D. degree in electrical and computer engineering from University of Illinois at Urbana-Champaign.

Dr. Chen's professional career spans academia and industry. He has held various R\&D management and engineering positions with companies in the USA over a period of 17 years, including AT\&T Bell Labs, Rockwell Science Center, iVast, and Digital Island. He was a U.S. delegate for ISO and ITU standards committees and contributed to the development of many interactive multimedia technologies that are now part of the MPEG-4 and JPEG-2000 standards. He has been with the College of Electrical Engineering and Computer Science, National Taiwan University, since August 2003, where he is currently a Distinguished Professor. His research interests lie in the broad area of multimedia signal processing and communications.

Dr. Chen was an Associate Editor of IEEE Transactions on Circuits and Systems for Video Technology from 2004 to 2010, IEEE Transactions on Image Processing from 1992 to 1994, and Pattern Recognition from 1989 to 1999. He served as a Guest Editor for IEEE Transactions on Circuits and Systems for Video Technology in 1999, IEEE Transactions on Multimedia in 2011, IEEE Journal of Selected Topics in Signal Processing in 2014, and Springer Multimedia Tools and Applications in 2015. Currently, he serves as a member of the IEEE SPS Fellow Review Committee and the IEEE Fourier Award Committee.

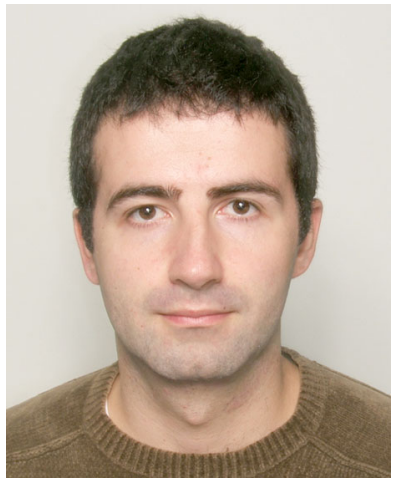

Dr. Velibor Adzic is the Director of Research and Development at Videopura LLC. Previously he worked as a visiting scholar in the Multimedia Lab at the Florida Atlantic University. He obtained PhD degree in Computer Science from Florida Atlantic University in 2014. His research interests are in video coding, computer vision, and image processing. 


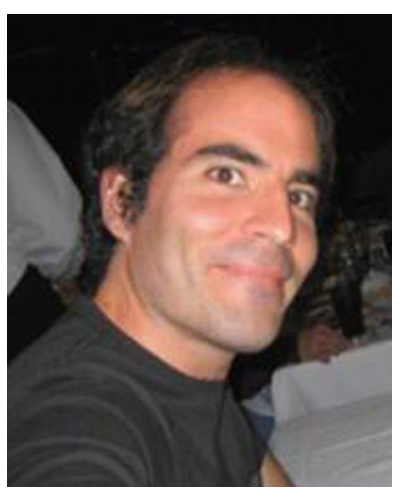

Gerardo Fernández-Escribano received the M.Sc. degree in Computer Engineering and the Ph.D. degree from the University of Castilla-La Mancha (UCLM), Albacete, Spain, in 2003 and 2007, respectively. In 2008, he joined the Department of Computer Systems at the UCLM, where he is currently an Associate Ph.D. Professor at the School of Industrial Engineering. His research interests include multimedia standards, video transcoding, video compression, video transmission, and machine learning mechanisms. He has also been a visiting researcher at the Florida Atlantic University, Boca Raton (USA), and at the Friedrich Alexander Universität, ErlangenNuremberg (Germany). 